\title{
Imitadores del ictus: un reto para el médico de urgencias
}

\section{Stroke mimics: a challenge for the emergency physician}

\author{
J. Valle ${ }^{1}$, E. Lopera ${ }^{1}$, M Guillán ${ }^{2}$, MC Muñoz ${ }^{3}$, A. Sánchez ${ }^{1}$, Y. Hernández ${ }^{1}$
}

\section{RESUMEN}

Fundamento. Estudiar el número de pacientes diagnosticados de ictus en el servicio de urgencias hospitalario de un hospital de primer nivel y la proporción de estos pacientes que finalmente eran pseudoictus, así como describir y analizar las variables que pueden ayudar en el diagnóstico diferencial en los servicios de urgencias hospitalarios.

Método. Estudio prospectivo de 9 meses. Se incluyeron todos los pacientes valorados en el servicio de Urgencias e ingresados con el diagnóstico de ictus. Se analizaron diversas variables clínicas que incluían la historia previa, la historia del evento actual, el examen físico general y neurológico y la clasificación del NIHSS y de la Oxforshire Community Stroke. El diagnóstico final se realizó por un panel de expertos con acceso a las características clínicas, estudios de imagen y otras pruebas. El análisis univariante y multivariado determinó las características que ayudan a distinguir los ictus de los pseudoictus.

Resultados. Se registraron 144 casos de ictus; la muestra final la conformó 140 pacientes. El diagnóstico final fue de ictus en 103 de $140(73,6 \%)$ y de pseudoictus en el resto. Once variables predecían el diagnóstico en pacientes que se presentaban con sospecha de ictus: la edad superior a 70 años $(\mathrm{p}=0,012)$, una clasificación del NIHSS > 5, lograr una clasificación clínica $(\mathrm{p}=0,019)$ capaz de determinar el comienzo exacto $(\mathrm{p}=0,000)$, hallazgos vasculares anormales $(\mathrm{p}=0,014)$, desviación de la mirada $(\mathrm{p}=0.042)$, pérdida de la visión $(\mathrm{p}=0,052)$ y reflejo plantar extensor $(\mathrm{p}=0,025)$ favorecían el diagnóstico de ictus, mientras que las crisis comiciales ( $\mathrm{p}=0,029)$, los síntomas neurológicos no congruentes con el territorio vascular $(\mathrm{p}=0,022)$ y los hallazgos anormales en otros sistemas $(\mathrm{p}=0,14)$ favorecían el diagnostico de pseudoictus.

Conclusión. Los pseudoictus constituyeron un tercio de los pacientes ingresados desde el servicio de Urgencias como ictus siendo la etiología muy variada. Lograr una historia clínica adecuada y un examen físico preciso es de gran importancia y puede ayudar a facultativos menos experimentados.

Palabras claves. Pseudoictus. Ictus. Diagnóstico.

\begin{abstract}
Background. To study the number of patients diagnosed with a stroke in the emergency service of a first level hospital and the proportion of these patients who were finally stroke mimics, as well as to describe and analyze the variables that can help in differential diagnosis in hospital emergency services.
\end{abstract}

Method. Nine month prospective study. All patients evaluated in emergency services and admitted with a diagnosis of stroke were included. Different clinical variables were analyzed that included prior history, history of the current event, genera physical and neurological examination, NIHSS classification and Oxfordshire Community Stroke Project Classification The final diagnosis was made by a panel of experts with access to clinical characteristics, image studies and other tests. The univariate and multivariate analysis determined the characteristics that help in distinguishing strokes from stroke mimics.

Results. One hundred and forty-four cases of stroke were registered; the final sample was made up of 140 patients. The final diagnosis was stroke in 103 out of 140 (73.6\%) and stroke mimic in the rest. Eleven variables predicted the diagnosis in patients with a suspected stroke: age over 70 years $(\mathrm{p}=0.012)$, NIHSS classification $>5$, reaching a clinical classification $(\mathrm{p}=0.019)$ capable of determining the exact start $(\mathrm{p}=0.000)$, abnormal vascular findings $(\mathrm{p}=0.014)$, gaze deviation $(\mathrm{p}=0.042)$, sight loss $(\mathrm{p}=0.052)$ and extensor plantar response $(\mathrm{p}=0.025)$ favored diagnosis of stroke, while epileptic seizures $(\mathrm{p}=0.029)$, neurological symptoms not congruent with the vascular territory $(\mathrm{p}=0.022)$ and abnormal findings in other systems $(\mathrm{p}=0.14)$ favored diagnosis of stroke mimic.

Conclusion. Stroke mimics constitute one-third of the patients admitted from emergency services as strokes, with a highly varied etiology. Achieving a suitable clinical history and a precise physical examination is of great importance and can help less experienced doctors.

Key words. Stroke mimic. Stroke. Diagnosis.
1. Servicio de Urgencias y Cuidados Críticos. Hospital Valle de los Pedroches. Córdoba. España

2. Servicio de Neurología, Unidad de Ictus. Hospital Universitario Ramón y Cajal, Madrid. España

3. Departamento de Bioestadística y Metodología. IMIBIC. Hospital Reina Sofía. Córdoba. España.

Recepción: 13 de diciembre de 2013

Aceptación provisional: 13 de enero de 2014

Aceptación definitiva: 16 de enero de 2014

\section{Correspondencia:}

Joaquín Valle Alonso

Hospital Valle de los Pedroches

Servicio de Urgencias y Cuidados Críticos

C/ Juan del Rey Calero, s/n

14400 Pozoblanco,

Córdoba España

E-mail: joa51274@hotmail.com 


\section{INTRODUCCIÓN}

La patología cerebrovascular es la primera causa de mortalidad en la mujer y la segunda causa global en España. El ictus se produce por un trastorno circulatorio que altera transitoria o definitivamente el funcionamiento de una o varias partes del encéfalo. En la mayoría de los casos se presenta como un déficit neurológico focal brusco relacionado con un territorio vascular siendo sencillo de identificar. Pero existen patologías cuya presentación puede asemejarse a la del ictus y su diagnóstico diferencial es un reto en el servicio de urgencias, incluso con las nuevas técnicas de neuroimagen. Estas patologías que se presentan simulando un ictus pero que tras estudio se demuestra su diferente etiología son conocidas como imitadoras del ictus o pseudoictus (PI). En los servicios de urgencias hospitalarios (SUH) los PI representan de un 20 a un $30 \%$ de los pacientes ingresados con diagnóstico de ictus en un primer momento $^{1}$, así como de un 1,4 a un $15 \%$ de los pacientes que reciben trombolisis con activador recombinante del plasminógeno tisular (rt-PA iv) tienen un diagnóstico final de $\mathrm{PI}^{2-9}$.

La etiología es muy variada e incluye hematoma subdural, migraña, crisis comiciales, tumores, infecciones, esclerosis múltiple, trastornos somatomorfo ${ }^{10}$, delirio, etc ${ }^{1-9,11-17}$. Estudios recientes han identificado las características clínicas y radiológicas asociadas a un diagnóstico final de PI incluyendo signos vitales, síntomas clínicos, signos neurológicos, gravedad del ictus e incluso las secuelas neurológicas al alta 2,4,5,11,15,18. El urgenciólogo debe determinar si el déficit neurológico agudo representa un infarto cerebral u otra entidad. Reconocer rápidamente un ictus es de gran importancia; cuanto más rápido se administra el tratamiento revascularizador ${ }^{19} \mathrm{o}$ se investiga y se comienza la prevención secundaria para evitar nuevos eventos $^{20}$ el pronóstico es mejor. La dificultad comienza con el tipo de servicio de urgencias y la disponibilidad de recursos. Los hospitales terciarios tienen acceso inmediato a un neurólogo así como a un ra- diólogo para interpretar la neuroimagen. El neurólogo decide si se necesitan más pruebas complementarias así como la técnica de reperfusión óptima. En los hospitales comarcales así como los hospitales de alta resolución (HARE), se carece de neurólogo y es el médico de urgencias quien debe decidir el manejo del paciente y de ahí la importancia de diferenciar correctamente un PI.

El objetivo primario de nuestro trabajo fue estudiar el número de pacientes diagnosticados de ictus en un servicio de urgencias de un hospital de primer nivel (Hospital Valle de los Pedroches) y la proporción de pacientes de éstos que finalmente eran PI. Como objetivo secundario describir y analizar qué variables de estos dos grupos (ictus y PI) pueden ayudar en el diagnóstico diferencial, por su mayor asociación con un grupo u otro.

\section{MATERIAL Y MÉTODOS}

Se realizó un estudio observacional prospectivo de los pacientes que son valorados en el servicio de Urgencias e ingresados en el servicio de Medicina Interna con diagnóstico de ictus durante el periodo comprendido entre el 1 de enero y el 30 de septiembre de 2010. El estudio se realizó en un hospital comarcal con un servicio de urgencias que atiende 30.000 urgencias anuales con disponibilidad de pruebas complementarias (tomografía axial computarizada [TAC], resonancia magnética nuclear [RMN], ecografía doppler de carótidas, etc.). La Organización Mundial de la Salud (OMS) recomienda como definición para el accidente cerebrovascular "afección neurológica focal (o a veces general) de aparición súbita, que perdura más de 24 horas (o causa la muerte) y de presunto origen vascular". El accidente isquémico transitorio (AIT) lo define como "la presencia de síntomas neurológicos focales, pero con una duración inferior a 24 horas". Además, se puntualiza que el diagnóstico es clínico y no se basa en los resultados radiológicos ${ }^{21}$. Se estudiaron todos los pacientes con 
sospecha de ictus; la hemorragia subaracnoidea no se consideró como causa de ictus en este estudio. La fuente primaria de pacientes se obtuvo del servicio de Urgencias donde ingresaron el 97\% de los pacientes con ictus. Un grupo de facultativos de urgencias con un mínimo de experiencia de 5 años realizó una historia que incluía:

1. Historia médica previa incluyendo factores de riesgo vascular, AIT o ictus previo, factores de riesgo para un simulador de ictus (deterioro cognitivo, migraña, epilepsia, neoplasia y trastornos psicológicos).

2. Historia del evento actual.

3. Examen físico general.

4. Examen neurológico incluyendo la escala NIHSS.
5. La formulación diagnóstica que incluía la subclasificación de la Oxforshire Community Stroke (Bamford y (ol) $)^{22}$.

Para determinar el diagnóstico final se realizó en consenso con el grupo de medicina interna y facultativos de urgencias con especial dedicación a patología cerebrovascular con una análisis exhaustivo caso a caso. Tomando en cuenta las bases metodológicas del estudio Iberictus ${ }^{23}$ y en función de la información clínica disponible, los diagnósticos finales se clasificaron, según el grado de evidencia disponible, en seis posibles categorías. Se dicotomizó el diagnóstico final en ictus o pseudoictus para permitir el análisis de las características clínicas que permiten distinguir ambas condiciones (Tabla 1).

Tabla 1. Niveles de certeza diagnóstica

\begin{tabular}{|c|c|}
\hline Ictus & Pseudoictus \\
\hline $\begin{array}{l}\text { Ictus definido en el caso que cumple los crite- } \\
\text { rios de la OMS y no existen dudas razonables } \\
\text { en la valoración de la historia o documentación } \\
\text { relativa al caso sobre el origen cerebrovascular } \\
\text { del episodio vascular cerebral }\end{array}$ & $\begin{array}{l}\text { Un ictus no definido cuando las característi- } \\
\text { cas clínicas no sugerían una etiología vascular } \\
\text { y existía otra explicación convincente para los } \\
\text { síntomas presentes }\end{array}$ \\
\hline $\begin{array}{l}\text { AIT definido cuando se cumplen los criterios } \\
\text { de ictus, pero la sintomatología cede completa- } \\
\text { mente en menos de } 24 \text { horas. Un ictus posible } \\
\text { tenía características clínicas menos convincen- } \\
\text { tes pero podía tener presente una alternativa } \\
\text { diagnóstica y no existían pruebas definitivas de } \\
\text { un ictus }\end{array}$ & $\begin{array}{l}\text { Ictus posible tenía características clínicas me- } \\
\text { nos convincentes pero podía tener presente } \\
\text { una alternativa diagnóstica y no existían prue- } \\
\text { bas definitivas de un ictus }\end{array}$ \\
\hline $\begin{array}{l}\text { AIT definido cuando se cumplen los criterios } \\
\text { de ictus, pero la sintomatología cede completa- } \\
\text { mente en menos de } 24 \text { horas }\end{array}$ & $\begin{array}{l}\text { AIT posible cuando se cumplen los criterios de } \\
\text { ictus posible, pero la sintomatología no cede } \\
\text { completamente en menos de } 24 \text { horas }\end{array}$ \\
\hline
\end{tabular}

Se realizaron como exploraciones básicas en todos los casos análisis de sangre, electrocardiograma (ECG) y radiografía de tórax y como estudio de ictus una tomografía axial computarizada (TAC) craneal, ecocardiograma, eco-Doppler carotídeo y resonancia magnética craneal (RMN) como pruebas complementarias para corroborar los diagnósticos finales.
Se realizó un análisis descriptivo para las variables cualitativas con recuentos (n) y proporciones (\%), y para las variables cuantitativas se calcularon media (m), desviación stándar (DS), mediana (me), rango intercuartílico (RIC) y los valores mínimo (mín) y máximo (máx). Se determinó la bondad de ajuste a una distribución normal mediante la prueba de Shapiro-Wilk. Entre am- 
bos grupos (ictus y PI), se compararon los valores promedios mediante prueba $U$ de Mann-Whitney y las proporciones mediante pruebas ji-cuadrado $(\chi 2)$ para tablas de contingencia; en el caso de tablas 2 x 2 se utilizó el estadístico $\chi 2$ con corrección de Yates, y cuando alguna frecuencia esperada fue menor o igual a 5 se aplicó la prueba exacta de Fisher. Para conocer los factores asociados con la aparicion de ACVA se realizó un análisis de regresión logística múltiple y se calcularon odds ratio (OR) y sus intervalos de confianza correspondientes (IC 95\% de la OR). El análisis estadístico se realizó con el programa PASW Statistics 18. Todos los contrastes de hipótesis fueron bilaterales y se consideraron valores estadísticamente significativos aquéllos cuyo nivel de confianza fue superior al 95\% $(\mathrm{p}<0,05)$.

\section{RESULTADOS}

Se incluyeron 144 pacientes que se presentaron con cuadro sugestivo de ictus en el servicio de Urgencias; de éstos, 3 presentaron el episodio en 2 ocasiones y 1 paciente en 3 ocasiones, consideran-

Tabla 2. Características basales de la población estudiada dividida en grupos de ictus y pseudoictus.

\begin{tabular}{|c|c|c|c|}
\hline & $\begin{array}{c}\text { Total } \\
N=140\end{array}$ & $\begin{array}{c}\text { Pseudoictus } \\
\quad \mathbf{n}=\mathbf{3 7}\end{array}$ & $\begin{array}{c}\text { Ictus } \\
\mathbf{n}=103\end{array}$ \\
\hline Edad & $74 \pm 16(17-96)$ & $65 \pm 23(17-94)$ & $79 \pm 13(35-96)$ \\
\hline Sexo (mujer) & $68 / 140(48,6 \%)$ & $22 / 37(59,5 \%)$ & $46 / 103(44,7 \%)$ \\
\hline Factores de riesgo vascular * & $121 / 140(86,4 \%)$ & $28 / 37(75,7 \%)$ & $93 / 103(90,3 \%)$ \\
\hline Historia previa de ictus/AIT & $36 / 140(25,7 \%)$ & $11 / 37(29,7 \%)$ & $25 / 103(24,3 \%)$ \\
\hline Hábitos tóxicos (fumador, bebedor, otros) & $68 / 140(48,6 \%)$ & $19 / 37(51,4 \%)$ & $49 / 103(47,6 \%)$ \\
\hline Factores de riesgo de simuladores** & $76 / 140(54,3 \%)$ & $17 / 37(45,9 \%)$ & $50 / 103(48,5 \%)$ \\
\hline Escala de Rankin Dependiente & $46 / 140(32,9 \%)$ & $9 / 37(24,3 \%)$ & $37 / 103(35,9 \%)$ \\
\hline \multicolumn{4}{|l|}{ Tiempo de evolución desde el inicio de la clínica } \\
\hline$<1$ hora & $24 / 140(17,1 \%)$ & $5 / 37(13,5 \%)$ & $19 / 103(18,4 \%)$ \\
\hline 1-4 horas & $64 / 140(45,7 \%)$ & $20 / 37(54,1 \%)$ & $44 / 103(42,7 \%)$ \\
\hline 5-8 horas & $34 / 140(24,3 \%)$ & $6 / 37(16,2 \%)$ & $28 / 103(27,2 \%)$ \\
\hline $9-12$ horas & $5 / 140(3,6 \%)$ & $3 / 37(8,1 \%)$ & $2 / 103(1,9 \%)$ \\
\hline $12-24$ horas & $4 / 140(2,9 \%)$ & 0 & $4 / 103(3,9 \%)$ \\
\hline$>24$ horas & $9 / 140(6,4 \%)$ & $3 / 37(8,1 \%)$ & $6 / 103(5,8 \%)$ \\
\hline \multicolumn{4}{|l|}{ Clasificación escala de NIHHSS } \\
\hline $1-5$ puntos & $39 / 140(27,9 \%)$ & $20 / 37(54,1 \%)$ & $19 / 103(18,4 \%)$ \\
\hline 5-10 puntos & $57 / 140(40,7 \%)$ & $14 / 37(37,8 \%)$ & $43 / 103(41,8 \%)$ \\
\hline 10-25 puntos & $27 / 140(19,3 \%)$ & $3 / 37(8,1 \%)$ & $24 / 103(23,3 \%)$ \\
\hline$>25$ puntos & $17 / 140(12,1 \%)$ & 0 & $17 / 103(16,5 \%)$ \\
\hline \multicolumn{4}{|l|}{ Clasificación clínica } \\
\hline Infarto total de la circulación anterior o TACI & $21 / 140(15 \%)$ & $2 / 37(5,4 \%)$ & $19 / 103(18,5 \%)$ \\
\hline Infarto parcial de la circulación anterior o PACI & $18 / 140(12,9 \%)$ & $3 / 37(8,1 \%)$ & $15 / 103(14,6 \%)$ \\
\hline Infarto lacunar o LACI & $21 / 140(15 \%)$ & $2 / 37(5,4 \%)$ & $19 / 103(18,5 \%)$ \\
\hline Infarto en la circulación posterior o POCI & $24 / 140(17,1 \%)$ & $6 / 37(16,2 \%)$ & $18 / 103(17,5 \%)$ \\
\hline Afasia global sin hemiparesia & $11 / 140(7,9 \%)$ & $1 / 37(2,7 \%)$ & $10 / 103(9,7 \%)$ \\
\hline No seguro & $40 / 140(28,6 \%)$ & $21 / 37(56,8 \%)$ & $19 / 103(18,5 \%)$ \\
\hline
\end{tabular}

\footnotetext{
* Hipertensión arterial, diabetes mellitus, cardiopatía isquémica, fibrilación auricular, arteriopatía periférica.

** Deterioro cognitivo, migraña, epilepsia, neoplasia conocida, trastorno psicológico.
} 
do para el estudio uno de los episodios de cada paciente. La muestra final fue de 140 pacientes. El diagnóstico final determinado por el panel de consenso determinó como ictus definido 63 (45\%), AIT definido $26(18,6 \%)$ e ictus probable $14(10 \%)$ con un total de 103 de 140 (73,6\%). Se encontró como ictus no definido $25(17,9 \%)$, ictus posible $8(5,7 \%)$ y AIT posible $4(2,9 \%)$. representado los SI ( $n=37$ ) un $26,4 \%$. Se diagnosticaron 12 ictus hemorrágicos $(11,7 \%)$ del total de ictus.
En la tabla 2 se representan las características clínicas de los pacientes divididos en ictus y PI. En la tabla 3 se representan las causas de PI; destacan las tóxico/metabólicas que incluyen presentaciones como la hipoglucemia, la hiperglucemia con situación hiperosmolar, la hipernatremia y la encefalopatía hepática, en el caso de la sepsis como causa de PI la etiología más frecuente fue la neumonía. En el resto de diagnósticos hay que destacar que muchos son de etiología neurológica.

Tabla 3. Etiología de los pseudoictus

\begin{tabular}{lc}
\hline \multicolumn{1}{c}{ Causas pseudoictus } & Número (\%) \\
\hline Tóxico/metabólicas & $7(18,9)$ \\
\hline Síndrome confusional agudo & $5(13,5)$ \\
\hline Crisis comicial y parálisis de Todd & $5(13,5)$ \\
\hline Lesión ocupante de espacio & $3(8,1)$ \\
\hline Sepsis & $3(8,1)$ \\
\hline Síncope/presíncope & $3(8,1)$ \\
\hline Trastorno somatomorfo & $2(5,4)$ \\
\hline Migraña & $2(5,4)$ \\
\hline Disfunción vestibular aguda & $1(2,7)$ \\
\hline Neuropatías craneales & $1(2,7)$ \\
\hline Síndrome vasoconstricción cerebral reversible & $1(2,7)$ \\
\hline Síndrome de HaNDL $($ Headache and Neurological & $1(2,7)$ \\
Deficit and Lymphocytosis) & $1(2,7)$ \\
\hline Emergencia hipertensiva & $1(2,7)$ \\
\hline Síndrome Guillain-Barré & $1(2,7)$ \\
\hline Esclerosis múltiple &
\end{tabular}

En las tablas 4 y 5 se muestra el análisis univariado señalando las relaciones más importantes. Un ictus era más probable si se era capaz de determinar el tiempo exacto del comienzo de la sintomatología bien definida $(\mathrm{p}<0,001)$, una edad mayor de 70 años ( $\mathrm{p} \leq 0,001)$, una Escala NIHSS (National Institute of Health Stroke Scale) $>5$ puntos $(\mathrm{p}=0,002)$, síntomas claros de lateralización presentes $(<0,001)$, si se lograba clasificar el evento neurológico en la clasificación de la Oxfordshire Community Stroke Project $(\mathrm{p}<0,001)$ sobre todo en los de infarto total de la circulación anterior (TACI) e Infarto parcial de la cir- culación anterior (PACI), no así en los casos de infarto en la circulación posterior (POCI), así como la presencia factores de riesgo vascular $(p=0,031)$. En el caso de los PI era más probable su presentación si existían hallazgos anormales en otros sistemas, especialmente síntomas respiratorios (ejemplo, crepitantes) o abdominales $(p=0,070)$, estar inconsciente a su llegada a urgencias $(p=0,252)$, ser mujer $(p=0,125)$, y los factores de riesgo clásicos para los PI (deterioro cognitivo, migraña, epilepsia, neoplasia conocida y trastornos psicológi$\cos )(\mathrm{p}=0,786)$ y un tiempo de evolución de más de 8 horas $(\mathrm{p}=0,68)$ 
Tabla 4. Características de la población estudiada (global y por grupos)

\begin{tabular}{|c|c|c|c|c|c|c|}
\hline & $\begin{array}{c}\text { Total } \\
N=140\end{array}$ & $\begin{array}{c}\text { Pseudoictus } \\
\mathbf{n}=\mathbf{3 7}\end{array}$ & $\begin{array}{c}\text { ictus } \\
n=103\end{array}$ & $P^{*}$ & OR & $\begin{array}{c}\text { IC } 95 \% \\
\text { de la OR }\end{array}$ \\
\hline $\begin{array}{l}\mathrm{m} \pm \mathrm{DS} \\
\mathrm{me} \pm \mathrm{RIC} \\
\text { mín-máx }\end{array}$ & $\begin{array}{c}74 \pm 16 \\
79 \pm 15 \\
(17-96)\end{array}$ & $\begin{array}{c}65 \pm 23 \\
74 \pm 38 \\
(17-94)\end{array}$ & $\begin{array}{c}77 \pm 11 \\
79 \pm 13 \\
(35-96)\end{array}$ & $<0,001$ & 1,04 & $1,02-1,07$ \\
\hline Edad superior a 70 años & $104(74,3 \%)$ & $21(56,8 \%)$ & $83(80,6 \%)$ & & & \\
\hline Género (mujer) & $68(48,6 \%)$ & $22(59,5 \%)$ & $46(44,7 \%)$ & 0,125 & 0,55 & $0,26-1,18$ \\
\hline FR vascular & $121(86,4 \%)$ & $28(75,7 \%)$ & $93(90,3 \%)$ & 0,031 & 2,99 & $1,11-8,08$ \\
\hline ACV/AIT previo & $36(25,7 \%)$ & $11(29,7 \%)$ & $25(24,3 \%)$ & 0,515 & 0,76 & $0,33-1,75$ \\
\hline Antecedentes de hábitos tóxicos & $68(48,6 \%)$ & $19(51,4 \%)$ & $49(47,6 \%)$ & 0,693 & 0,86 & $0,41-1,82$ \\
\hline Tto previo con antiagregantes & $88(62,9 \%)$ & $22(59,5 \%)$ & $66(64,1 \%)$ & 0,618 & 1,22 & $0,56-2,63$ \\
\hline Tto previo antihipertensivos & $89(63,6 \%)$ & $19(54,3 \%)$ & $70(68,0 \%)$ & 0,147 & 1,79 & $0,82-3,91$ \\
\hline FR para pesudoictus & $67(47,9 \%)$ & $17(45,9 \%)$ & $50(48,5 \%)$ & 0,786 & 1,11 & $0,52-2,36$ \\
\hline Tto previo hipolipemiantes & $56(40,3 \%)$ & $12(33,3 \%)$ & $44(42,7 \%)$ & 0,325 & 1,49 & $0,67-3,30$ \\
\hline Tto previo antiarritmicos & $62(44,3 \%)$ & $17(45,9 \%)$ & $45(43,7 \%)$ & 0,813 & 0,91 & $0,43-1,94$ \\
\hline Escala Rankin (dependiente) & $46(32,9 \%)$ & $9(24,3 \%)$ & $37(35,9 \%)$ & 0,201 & 1,74 & $0,74-4,09$ \\
\hline Inconsciente a la llegada & $39(27,9 \%)$ & $13(35,1 \%)$ & $26(25,2 \%)$ & 0,252 & 0,62 & $0,28-1,40$ \\
\hline Escala Glasgow $<8$ puntos & $32(22,9 \%)$ & $7(18,9 \%)$ & $25(24,3 \%)$ & 0,507 & 1,37 & $0,54-3,51$ \\
\hline TAS > $160 \mathrm{~mm} \mathrm{Hg}$ a la llegada & $54(38,6 \%)$ & $15(40,5 \%)$ & $39(37,9 \%)$ & 0,774 & 0,89 & $0,42-1,93$ \\
\hline $\mathrm{FC}>100 \mathrm{lpm}$ a la llegada & $32(22,9 \%)$ & $7(18,9 \%)$ & $25(24,3 \%)$ & 0,507 & 1,37 & $0,54-3,51$ \\
\hline Hallazgos anormales en otros sistemas* & $21(15,0 \%)$ & $9(24,3 \%)$ & $12(11,7 \%)$ & 0,070 & 0,41 & $0,16-1,07$ \\
\hline Glucemia $>140 \mathrm{mg} / \mathrm{dl}$ a la llegada & $58(41,4 \%)$ & $15(40,5 \%)$ & $43(41,7 \%)$ & 0,898 & 1,05 & $0,49-2,26$ \\
\hline SatO2 > 93\% a la llegada & $96(68,6 \%)$ & $26(70,3 \%)$ & $70(68,0 \%)$ & 0,795 & 0,90 & $0,40-2,03$ \\
\hline Lateralización presente & $59(42,1 \%)$ & $6(16,2 \%)$ & $53(51,5 \%)$ & $<0,001$ & 5,48 & $2,11-14,24$ \\
\hline Tiempo evolución $>8 \mathrm{~h}$ & $18(12,9 \%)$ & $6(16,2 \%)$ & $12(11,7 \%)$ & 0,382 & 0,68 & $0,24-1,97$ \\
\hline Escala NIHSS > 5 puntos & $44(31,4 \%)$ & $3(8,1 \%)$ & $41(39,8 \%)$ & 0,002 & 7,50 & $2,16-26,02$ \\
\hline Clasificación clínica realizada & $95(67,9 \%)$ & $14(37,8 \%)$ & $81(78,6 \%)$ & $<0,001$ & 6,05 & $2,68-13,66$ \\
\hline Capaz de determinar el comienzo exacto & $105(75,2 \%)$ & $13(36,1 \%)$ & $92(89,3 \%)$ & $<0,001$ & 14,80 & $5,87-37,27$ \\
\hline
\end{tabular}

Los datos se expresan como recuentos (n) y porcentajes (\%), excepto para la variable edad cuando se considera cuantitativa continua $(\mathrm{m}=$ media, $\mathrm{DS}=$ desviación standar, $\mathrm{me}=$ mediana, $\mathrm{RIC}=$ rango intercuartílico, mín $=$ mínimo, máx = máximo).

FR= factores de riesgo; ACVA/AIT = Accidente cerebrovascular agudo, accidente isquémico transitorio; Tto= tratamiento; TAS= tensión arterial sistólica; FC= frecuencia cardiaca; SatO2=saturación arterial de oxígeno; NIHSS= National institute of Health Stroke Scale; * $\mathrm{p}$ = nivel de significación para el estadístico de Wald; OR = Odds ratio obtenidas con análisis de regresión logística simple. IC = Intervalo de confianza del 95\%; *síntomas respiratorios, abdominales u otros. 
Tabla 5. Síntomas y signos en los pacientes estudiados (global y por grupos según el diagnóstico final)

\begin{tabular}{|c|c|c|c|c|c|c|}
\hline & $\begin{array}{c}\text { Total } \\
N=140\end{array}$ & $\begin{array}{c}\text { pseudoictus } \\
\mathbf{n}=37\end{array}$ & $\begin{array}{c}\text { ictus } \\
\mathrm{n}=103\end{array}$ & $\mathbf{P}^{*}$ & OR & $\begin{array}{c}\text { IC } 95 \% \\
\text { de la OR }\end{array}$ \\
\hline Pérdida de conciencia & $22(15,7 \%)$ & $5(13,5 \%)$ & $17(16,5 \%)$ & 0,869 & 1,26 & $0,43-3,71$ \\
\hline Cefalea & $6(4,3 \%)$ & $3(8,1 \%)$ & $3(2,9 \%)$ & 0,188 & 0,34 & $0,06-1,76$ \\
\hline Crisis comicial al comienzo & $8(5,7 \%)$ & $5(13,5 \%)$ & $3(2,9 \%)$ & 0,030 & 0,19 & $0,04-0,85$ \\
\hline Hallazgos vasculares anormales ${ }^{\underline{a}}$ & $28(20,0 \%)$ & $1(2,7 \%)$ & $27(26,2 \%)$ & 0,005 & 12,79 & $1,67-97,86$ \\
\hline $\begin{array}{l}\text { Síntomas neurológicos no } \\
\text { congruentes con territorio vascular }\end{array}$ & $57(40,7 \%)$ & $21(56,8 \%)$ & $36(35,0 \%)$ & 0,034 & 0,41 & $0,19-0,88$ \\
\hline Pérdida sensitiva cara & $9(6,4 \%)$ & $1(2,7 \%)$ & $8(7,8 \%)$ & 0,445 & 3,03 & $0,37-25,10$ \\
\hline Debilidad brazo & $8(5,7 \%)$ & $2(5,4 \%)$ & $6(5,8 \%)$ & 1,000 & 1,08 & $0,21-5,62$ \\
\hline Pérdida sensitiva brazo & $3(2,1 \%)$ & $1(2,7 \%)$ & $2(1,9 \%)$ & 1,000 & 0,71 & $0,06-8,10$ \\
\hline Debilidad de la mano & $9(6,4 \%)$ & $1(2,7 \%)$ & $8(7,8 \%)$ & 0,445 & 3,03 & $0,37-25,10$ \\
\hline Pérdida sensitiva mano & $5(3,6 \%)$ & $1(2,7 \%)$ & $4(3,9 \%)$ & 1,000 & 1,45 & $0,16-13,45$ \\
\hline Debilidad de la pierna & $14(10,0 \%)$ & $4(10,8 \%)$ & $10(9,7 \%)$ & 1,000 & 0,89 & $0,26-3,02$ \\
\hline Pérdida sensitiva pierna & $10(7,1 \%)$ & $2(5,4 \%)$ & $8(7,8 \%)$ & 1,000 & 1,47 & $0,30-7,28$ \\
\hline Vértigo/mareo & $13(9,3 \%)$ & $3(8,1 \%)$ & $10(9,7 \%)$ & 1,000 & 1,22 & $0,32-4,69$ \\
\hline Confusión & $6(4,3 \%)$ & $2(5,4 \%)$ & $4(3,9 \%)$ & 0,655 & 0,71 & $0,12-4,03$ \\
\hline Trastorno al habla & $18(12,9 \%)$ & $7(18,9 \%)$ & $11(10,7 \%)$ & 0,251 & 0,51 & $0,18-1,44$ \\
\hline Disartria/afasia & $41(29,3 \%)$ & $10(27 \%)$ & $31(30,1 \%)$ & 0,888 & 1,16 & $0,50-2,69$ \\
\hline Hemianopsia & $6(4,3 \%)$ & $2(5,4 \%)$ & $4(3,9 \%)$ & 0,655 & 0,71 & $0,12-4,03$ \\
\hline Inatención visual & $14(10,0 \%)$ & $2(5,4 \%)$ & $12(11,7 \%)$ & 0,355 & 2,31 & $0,49-10,84$ \\
\hline Asimetría facial & $24(17,1 \%)$ & 0 & $24(23,3 \%)$ & 0,003 & $\ldots$ & $\ldots-\ldots$ \\
\hline Desviación de la mirada & $24(17,1 \%)$ & $2(5,4 \%)$ & $22(21,4 \%)$ & 0,051 & 4,75 & $1,06-21,32$ \\
\hline Debilidad brazo/mano & $21(15,0 \%)$ & $3(8,1 \%)$ & $18(17,5 \%)$ & 0,271 & 2,40 & $0,66-8,68$ \\
\hline Pérdida de visión & $19(13,6 \%)$ & $1(2,7 \%)$ & $18(17,5 \%)$ & 0,049 & 7,62 & $0,98-59,28$ \\
\hline Debilidad pierna & $11(7,9 \%)$ & $2(5,4 \%)$ & $9(8,7 \%)$ & 0,728 & 1,68 & $0,34-8,14$ \\
\hline Pérdida sensitiva & $6(4,3 \%)$ & $2(5,4 \%)$ & $4(3,9 \%)$ & 0,655 & 0,71 & $0,12-4,03$ \\
\hline Disfunción visuoespacial & $11(7,9 \%)$ & $2(5,4 \%)$ & $9(8,7 \%)$ & 0,728 & 1,68 & $0,34-8,14$ \\
\hline Ataxia miembro inferior & $10(7,1 \%)$ & $1(2,7 \%)$ & $9(8,7 \%)$ & 0,291 & 3,45 & $0,42-28,19$ \\
\hline Reflejo plantar extensor & $24(17,1 \%)$ & $1(2,7 \%)$ & $23(22,3 \%)$ & 0,014 & 10,35 & $1,34-79,63$ \\
\hline
\end{tabular}

Los datos se expresan como recuentos (n) y porcentajes (\%). ${ }^{*} \mathrm{p}=$ nivel de significación estadística obtenido tras la comparación de proporciones mediante estadísticos ji-cuadrado. OR = Odds ratio calculada con tablas de contingencia. IC = Intervalo de confianza del 95\%. TA diastólica mayor de $150 \mathrm{mmHg}$ o diastólica mayor de $90 \mathrm{mmHg}$, fibrilación auricular, enfermedad valvular o arteriopatia periférica

En relación a los síntomas y signos de los pacientes estudiados los hallazgos vasculares anormales ( $\mathrm{p}=0,005)$, la desviación de la mirada $(\mathrm{p}=0,051)$, la pérdida de visión $(\mathrm{p}=0,049)$, la presencia de reflejo plantar extensor $(\mathrm{p}=0.014)$ así como la asimetría facial $(\mathrm{p}=0,003)$ son más probables en pacientes con ictus, mientras que la crisis comicial al comienzo $(\mathrm{p}=0,030)$ y los síntomas neurológicos no congruentes con territorio vascular $(\mathrm{p}=0,034)$ favorecían el diagnóstico de PI. 
Tabla 6. Síntomas y signos en los pacientes estudiados (global y por grupos según el diagnóstico final) Modelo final de regresión logística para la predicción del ictus

\begin{tabular}{|c|c|c|c|}
\hline Variable & $\mathbf{P}^{*}$ & OR & IC $95 \%$ de la OR \\
\hline Edad superior a 70 años & 0,012 & 1,04 & $1,009-1,074$ \\
\hline NIHSS $>5$ & 0,026 & 7,57 & $1,268-45,230$ \\
\hline Clasificación clínica & 0,019 & 4,05 & $1,257-13,071$ \\
\hline Capaz determinar tiempo exacto comienzo & 0,000 & 14,90 & $4,568-48,627$ \\
\hline Hallazgos vasculares anormales* & 0,014 & 12,79 & $1,67-97,86$ \\
\hline Desviación de la mirada & 0,042 & 4,75 & $1,06-21,32$ \\
\hline Pérdida de visión & 0,052 & 7,62 & $0,98-59,28$ \\
\hline Reflejo plantar extensor & 0,025 & 10,35 & $1,34-79,63$ \\
\hline Crisis comiciales & 0,029 & 0,19 & $0,04-0,85$ \\
\hline Síntomas neurológicos no congruentes con territorio vascular & 0,022 & 0,41 & $0,19-0,88$ \\
\hline Hallazgos anormales en otros sistemas ${ }^{\underline{a}}$ & 0,009 & 0,14 & $0,032-0,610$ \\
\hline
\end{tabular}

El modelo matemático predice la probabilidad de ictus (en un rango de 0 a 1). La ecuación matemática utiliza el coeficiente para cada variable mas una constante para calcular la probabilidad. El OR aporta el "peso" de la importancia de la variable (ej, si es capaz de determinar el comienzo exacto de los síntomas de focalización neurológica y la pérdida de visión al examen clínico son los factores predictivos más importantes). * TA diastólica mayor de 150 mmHg o diastólica mayor de $90 \mathrm{mmHg}$, fibrilación auricular, enfermedad valvular o arteriopatia periférica

${ }^{a}$ Respiratorios, abdominales u otros sistemas

Después de realizar el análisis multivariado se ha obtenido 11 items que ayudarían en el servicio de Urgencias a predecir independientemente el diagnóstico (Tabla 6).

\section{DISCUSIÓN}

En relación a la incidencia del ictus los datos son muy similares a un estudio realizado en el área norte sanitaria ${ }^{24}$ con una incidencia anual próxima a 138 pacientes al año y de 162/100.000 habitantes. Del estudio realizado se deduce que la incidencia del ictus en el área geográfica analizada está dentro de las cifras publicadas para España, que se sitúan entre los 125 y los 226 casos por 100.000 habitantes/ año, y los conocidos para Andalucía, que son 186/100.000 habitantes/año. Nuestros resultados son muy similares al estudio Iberictus $^{25}$ donde el $71,1 \%$ de los casos se obtuvieron del CMBD y el resto a través de los servicios de urgencias los resultados en cuanto a probabilidad diagnóstica de ictus fueron: el $31 \%$ AIT definido, el $60 \%$ ictus definidos y el $8,7 \%$ ictus posible con datos insuficientes; el 11,7\% fueron ictus hemorrágicos.
Los PI pueden constituir casi el $27 \%$ de todos los pacientes con un diagnóstico inicial de ictus en un hospital comarcal en el SUH. Estos datos concuerdan con el estudio de Moeller y $\mathrm{col}^{26}$, en el que se encontró una discrepancia de alrededor de un tercio de los casos en el diagnóstico de la patología neurológica entre el servicio de Urgencias y el diagnóstico final, principalmente por considerar ictus otras patologías, como crisis comiciales, síncope, vértigo, cefalea o síndromes psicógenos. En el estudio de Hand y col $^{15}$ se estimó en un $31 \%$ la frecuencia de PI, comparando el diagnóstico inicial a la cabecera del paciente con el diagnóstico final tras evaluación por un panel de expertos con acceso a las pruebas complementarias; fueron las crisis epilépticas, sepsis y alteraciones toxicometabólicas (principalmente hipoglucemia) la etiología de los SI.

En el reciente estudio de Whiteley y Gibson $^{1}$ se ha realizado un metaanálisis con una revisión de los estudios prospectivos para determinar la proporción de pacientes con ictus confirmado. Se identificaron 29 estudios con 8.839 pacientes, 13 estudios de servicios de urgencias, cinco 
de unidades de ictus o clínicas de AIT, tres de atención primaria, 3 de ambulancias y 5 no específicos. Aproximadamente tres cuartos (74\%) de los pacientes tenían un diagnóstico de ictus y un $26 \%$ de simuladores de ictus. Las cinco causas más frecuente de simuladores de ictus eran las crisis comiciales, sincope, sepsis, migraña y tumores cerebrales.

En un estudio nacional realizado en el servicio de urgencias del hospital Virgen del Rocío durante 3 meses $^{27}$ se analizaron un total de 358 pacientes: 110 AIT, 191 isquémicos y 57 hemorrágicos. Se seleccionaron 65 falsos diagnósticos, correspondientes al 18,2\% del total (el 41,8\% de los AIT) y al $31,8 \%$ de los ictus de alta en urgencias (el 46,4\% de los AIT). Los subtipos de falsos diagnósticos fueron: 46 AIT (70,8\%), 18 isquémicos $(27,7 \%)$ y uno hemorrágico $(1,5 \%)$. Los diagnósticos alternativos fueron: síncope/presíncope, síndrome confusional/desorientación, disminución del nivel de conciencia, debilidad generalizada, mareo/vértigo, disartria aislada, crisis epiléptica y otros. A diferencia de nuestro estudio los diagnósticos se evaluaron al alta incluyendo el alta de urgencias mientras que en el nuestro se realiza en pacientes hospitalizados y tras la evaluación de un panel de expertos.

Los últimos estudios en relación a PI se han realizados en los subgrupos de pacientes trombolizados. En estos casos el número real de pacientes que se presentan con un PI comparado con aquellos que tienen un ictus verdadero varía dependiendo del estudio de investigación relacionado con el tema. Un estudio prospectivo realizado en Alemania del 2004 al 2010 tenía a 42 de 648 (6.5\%) de los pacientes con sospecha de ictus isquémico y trombolizados con un diagnóstico final de PI. Solamente reportó 1 caso de reacciones adversas (edema orolingual $)^{28}$. Chernyshev y col${ }^{4}$ encontraron que un $14 \%$ de los pacientes tratados con trombolisis se diagnosticaron de PI y ninguno sufrió ninguna complicación. Este grupo de pacientes solamente incluía aquellas tratados en el periodo de ventana de 3 horas tras comenzar los síntomas. El estudio de Brunser y col$^{8}$ valora a 842 pacientes de los cuales $113(13,4 \%)$ se consideraron PI. Estos pacientes eran más jóvenes, presentaban mayor frecuencia de diabetes, llegaban tardíamente a urgencias, poseían menor puntuación en la escala de NIHSS, las causas más comunes eran trastornos tóxico-metabólicos $(30,1 \%)$ y crisis comiciales $(19,5 \%)$ y la mayoría de los pacientes con PI frecuentemente tenía criterios de exclusión por presentarse mas allá de la ventana de trombolisis establecida. En relación al estudio nacional de Guillan y col del hospital Universitario Ramón y Cajal ${ }^{7}$, se realiza un registro de los pacientes tratados con rt-PA iv en la unidad de ictus; de los 621 pacientes tratados solo $15(2,2 \%)$ eran PI. Estos pacientes eran jóvenes, tenían una puntuación menor en el score NIHSS, menores factores de riesgo vascular y predominaban los síntomas del hemisferio izquierdo. La afasia global sin hemiparesis era el síntoma de presentación en el 54\% de los pacientes con PI. La etiología era de trastornos somatomorfos (5), síndrome de HaNDL (cefalea con déficit neurológico y linfocitosis del LCR) (3), encefalitis herpética (2), tumores gliales (2) y migraña con aura, convulsiones focales y trombosis venosa central, concluyendo que el beneficio de la trombolisis supera el riesgo.

Un reciente estudio multicéntrico (9) identificó 100 pacientes con PI resultando en una frecuencia de 1,8\%. Estos pacientes eran jóvenes, mujeres y con menos factores de riesgo, excepto por ser fumadores y no poseer antecedentes de ictus o AIT. La incidencia de hemorragia intracraneal sintomática en los PI fue de un 1,0\% demostrando que en centros experimentados en el manejo del ictus los pacientes trombolizados solo muy pocos tenían un diagnóstico final diferente al ictus. La frecuencia de complicaciones en estos pacientes era muy baja. Se ha prestado poca atención a los pacientes y las condiciones que imitan los AIT. En un estudio de cohorte prospectivo de 2 años en Suiza, aproximadamente el $20 \%$ de los pacientes con sospecha de AIT en la presentación eran imitadores de AIT $^{29}$.

En el análisis univariante se han identificado varios factores que pueden ayudar 
a diferenciar entre ictus y PI. En el estudio de Libman y col $^{11}$ se encontró que el sexo femenino, campos visuales anormales, TA diastólica mayor de $90 \mathrm{~mm} \mathrm{Hg}$ y la presencia de fibrilación auricular incrementaba el riesgo de ictus. El estudio de Ferro y $\mathrm{col}^{30}$ encontró que los PI eran más probables en pacientes que no tenían factores de riesgo vascular aunque no pudieron confirmarlo. Los estudios más antiguos ${ }^{31,32}$ sugieren que la evolución temporal de los síntomas distinguía entre los eventos vasculares y no vasculares aunque esto beneficiaba poco en la práctica diaria.

En el estudio de Hand y col $^{15}$ se encontraron 8 predictores independientes para el diagnóstico en pacientes con ictus. El daño cognitivo conocido y los hallazgos anormales en otros sistemas favorecían el diagnóstico de PI mientras que el comienzo exacto de los síntomas, la historia definida de síntomas de focalización neurológica, cualquier hallazgo vascular anormal, la puntuación de NIHSS 1-4, NIHSS 5-10, NIHSS $>10$, la lateralización de los signos a la derecha o izquierda y una posible clasificación de la OCSP favorecían el diagnóstico de ictus. Los resultados de nuestro estudio son bastante similares a éstos, demostrando la importancia de los datos de la anamnesis y el examen físico en el servicio de urgencias en los pacientes con sospecha de ictus. Con un mejor conocimiento de las características claves que pueden distinguir entre el ictus y los PI y los médicos menos experimentados pueden tener nuevas herramientas para un mejor diagnóstico. El reciente estudio de Merino y $\mathrm{col}^{33}$, el mayor efectuado hasta la fecha de PI donde se evaluó de forma prospectiva los pacientes valorados por el programa de los institutos nacionales de salud de ictus en EEUU en 10 años, incluyó a 8.187 pacientes donde un 30\% tenía un diagnóstico final de PI. Los pacientes con PI eran más jóvenes y la proporción era mayor en las mujeres que no tenían factores de riesgo, llegaban al servicio de Urgencias en vehículos personales o tras la activación del código ictus así y el comienzo de los síntomas era intrahospitalario. Esta proporción era mayor en pacientes afroamericanos que en caucásicos y los factores de mayor riesgo para un PI eran carecer de una historia de hipertensión, fibrilación auricular o hiperlipidemia.

Nuestro estudio tiene varias limitaciones, así la valoración de los pacientes se ha realizado en el servicio de Urgencias y el panel de expertos para determinar el diagnóstico final no incluía neurólogos especializados en patología cerebrovascular, no obstante una de las razones del estudio era la valoración de estos pacientes por profesionales médicos del servicios de Urgencias. Los criterios de inclusión pueden haber sido muy estrictos y algunos pacientes dados de alta del servicio de Urgencias con diagnóstico de ictus no se han incluido en el estudio, no obstante el protocolo del hospital es de ingresar a todos los pacientes con diagnóstico de ictus para el estudio y tratamiento. Los valores pueden estar sesgados pues la muestra utilizada es tan solo la de aquellos pacientes que han sido hospitalizados, como sucede en muchos registros de ictus. Factores que pueden llevar a la confusión incluyen la diferencia de formación y experiencia de los facultativos de los servicios de urgencias. Finalmente existen problemas bien descritos con los modelos de regresión logística lo que implica que el modelo no debe ser generalizado a otras cohortes de pacientes.

En la actualidad, una prueba diagnóstica rápida y ampliamente disponible para el accidente cerebrovascular sigue siendo difícil de alcanzar. En un trabajo realizado en el hospital Vall d'Hebron ${ }^{34}$ se estudió el valor predictivo de un panel marcadores bioquímicos para el diagnóstico de enfermedad cerebrovascular (incluyendo los simuladores de ictus) donde se concluyó que una combinación de biomarcadores incluyendo caspasa-3 y D-dímero parece ser la más prometedora para lograr un rápido diagnóstico bioquímico de accidente cerebrovascular y este enfoque podría ser utilizado como una herramienta en el futuro en el diagnóstico preciso de pacientes con ictus agudo.

Del presente trabajo se puede concluir que los pseudoictus constituyeron un tercio 
de los pacientes ingresados desde el servicios de urgencias como ictus. La etiología fue muy variada destacando las causas tóxico/metabólicas, el síndrome confusional agudo y las crisis comiciales. En relación a las variable predictivas la edad superior a 70 años, una clasificación del NIHSS > 5, lograr una clasificación clínica, ser capaz de determinar el comienzo exacto de los síntomas, hallazgos vasculares anormales así como la desviación de la mirada, la pérdida de la visión y el reflejo plantar extensor favorecían el diagnóstico de ictus mientras que las crisis comiciales, los síntomas neurológicos no congruentes con el territorio vascular y los hallazgos anormales en otros sistemas favorecían el diagnóstico de PI.

\section{BIBLIOGRAFÍA}

1. GiBSon LM, Whiteley W. The differential diagnosis of stroke: a systematic review. J R Coll Physicians Edinb 2013; 4: 114-118.

2. Winkler DT, Fluri F, Fuhr P, Wetzel SG, Lyrer PA, Ruegg S, ET AL. Thrombolysis in stroke mimics: frequency, clinical characteristics, and outcome. Stroke 2009; 40: 1522-1525.

3. Uchino K, Massaro L, Hammer MD. Transient ischemic attack after tissue plasminogen activator: Aborted stroke or unnecessary stroke therapy? Cerebrovasc Dis. 2010; 29: 57-61.

4. Chernyshev OY, Martin-Schild S, Albright KC, Barreto A, Misra V, Acosta I, ET al. Safety of tPA in stroke mimics and neuroimaging-negative cerebral ischemia. Neurology 2010; 74: 13401345.

5. Giraldo EA, Khalid A, Zand R. Safety of intravenous thrombolysis within $4.5 \mathrm{~h}$ of symptom onset in patients with negative post-treatment stroke imaging for cerebral infarction. Neurocrit Care 2011; 15: 76-79

6. Tsivgoulis G, Alexandrov AV, Chang J, Sharma VK, Hoover SL, Lao AY, ET AL. Safety and outcomes of intravenous thrombolysis in stroke mimics: a 6-year, single-care center study and a pooled analysis of reported series. Stroke 2011; 42: 1771-1774.

7. Guillan M, Alonso-Canovas A, Gonzalez-Valcarcel J, Garcia Barragan N, Garcia Caldentey J, Hernandez-Medrano I, et al. Stroke mimics treated with thrombolysis: further evidence on safety and distinctive clinical features. Cerebrovasc Dis 2012; 34: 115-120.
8. Brunser AM, Illanes S, Lavados PM, Muñoz P, CÁrcamo D, Hoppe A et al. Exclusion criteria for intravenous thrombolysis in stroke mimics: an observational study. J Stroke Cerebrovasc Dis 2013; 22: 1140-1145.

9. ZinKSTOK SM, Engelter ST, Gensicke H, Lyrer PA, Ringleb PA, ARTTo V, ET AL. Safety of thrombolysis in stroke mimics: results from a multicenter cohort study. Stroke 2013 Apr; 44: 10801084.

10. Organización Mundial de la Salud (OMS). CIE-10. Décima revisión de la clasificación internacional de las enfermedades. Trastornos mentales y del comportamiento. Descripciones clínicas y pautas para el diagnóstico. División de Salud Mental, Ginebra. Meditor. Madrid 1992; 63-84.

11. LibMan RB, WirkowsKi E, Alvir J, RAO TH.Conditions that mimic stroke in the emergency department. Implications for acute stroke trials. Arch Neurol 1995; 52: 1119-1122.

12. Harbison J, Hossain O, Jenkinson D, Davis J, Louw SJ, FORD GA. Diagnostic accuracy of stroke referrals from primary care, emergency room physicians, and ambulance staff using the face arm speech test. Stroke. 2003; 34: 71-76.

13. Scott PA, Silbergleit R. Misdiagnosis of stroke in tissue plasminogen activator-treated patients: characteristics and outcomes. Ann Emerg Med 2003; 42: 611-618.

14. ShellhaAs RA, Smith SE, O’Tool E, Licht DJ, ICHORD RN. Mimics of childhood stroke: characteristics of a prospective cohort. Pediatrics 2006; 118: 704-709.

15. Hand PJ, Kwan J, Lindley RI, Dennis MS, WardLAW JM. Distinguishing between stroke and mimic at the bedside: the brain attack study Stroke 2006; 37: 769-775.

16. Hemmen TM, Meyer BC, McClean TL, Lyden PD. Identification of nonischemic stroke mimics among 411 code strokes at the University of California, San Diego, Stroke Center. J Stroke Cerebrovasc Dis 2008; 17: 23-25.

17. VRoomen PC, Buddingh MK, LuiJckx GJ, De KeYSER J. The incidence of stroke mimics among stroke department admissions in relation to age group. J J Stroke Cerebrovasc Dis 2008; 17: 418-422.

18. El Husseini N, Goldstein LB. "Code stroke": hospitalized versus emergency department patients. J Stroke Cerebrovasc Dis 2013; 22: 345-348.

19. Wardlaw JM, Murray V, Berge E, del Zoppo G, SANDERCOCK P, LINDLEY RL et al. Recombinant tissue plasminogen activator for acute ischaemic stroke: an updated systematic review and meta-analysis. Lancet 2012; 379 : 2364-2372. 
20. Rothwell PM, Giles MF, Chandratheva A, MarQuARDT L, GERAGhty O, Redgrave JN, et al. Early use of Existing Preventive Strategies for Stroke (EXPRESS) study. Effect of urgent treatment of transient ischaemic attack and minor stroke on early recurrent stroke (EXPRESS study): a prospective population based sequential comparison. Lancet 2007; 370: 1432-1442.

21. Organización Mundial de la Salud. Manual de la OMS para la vigilancia paso a paso de accidentes cerebrovasculares. URL: http://www. paho.org/Spanish/AD/DPC/NC/stepsstroke. Pdf. [17.10.2009].

22. Dennis MS, BAMford JM, SANDERCock PA, WarLow CP, et al. Incidence of transient ischemic attacks in Oxfordshire, England. Stroke 1989; 20: 333-339.

23. Díaz-Guzmán J, Egido-Herrero JA, Gabriel-Sánchez R, Barberà G, Fuentes B, Fernández-Pérez C, AbiLLEIRA S. Proyecto Ictus del Grupo de Estudio de Enfermedades Cerebrovasculares de la Sociedad Española de Neurología. Incidencia de ictus en España. Bases metodológicas del estudio Iberictus. Rev Neurol 2008; 47: 617-623.

24. García-de Lucas MD, Casas-Fernández de Tejerina JM, CARA-GARcía M. Acute cerebrovascular disease in the Northern Cordoba Health Area. Rev Neurol 2007; 44: 68-74.

25. Díaz-Guzmán J, Egido JA, Gabriel-Sánchez R, Barberá-Comes G, Fuentes-Gimeno B, FernándezPÉREZ C; IBERICTUS Study Investigators of the stroke project of the spanish cerebrovascular diseases study group. Stroke and transient ischemic attack incidence rate in Spain: The IBERICTUS Study. Cerebrovasc Dis 2012; 34: 272-281.

26. Moeller JJ, Kurniawan J, Gubitz GJ, Ross JA, Bhan $\mathrm{V}$ et al. Diagnostic accuracy of neurological problems in the emergency department. Can J Neurol Sci 2008; 35: 335-341.

27. de la Torre-Laviana FJ, Moniche-Alvarez F, Palomino-García A, Cayuela-Domínguez A, Vigil E, JiMÉNEZ MD. False diagnoses of strokes in emergency departments. Rev Neurol 2010; 50: 463-469.

28. Förster A, Griebe M, Wolf ME, Szabo K, HenneriCI MG, KERN R. How to identify stroke mimics in patients eligible for intravenous thrombolysis? J Neurol 2012; 259: 1347-1353.

29. Amort M, Fluri F, Schäfer J, WeissKopf F, KatAN M, Burow A, et al. Transient ischemic attack versus transient ischemic attack mimics: frequency, clinical characteristics, and outcome. Cerebrovasc Dis 2011; 32: 57-64.

30. Ferro JM, Pinto AN, Falcão I, Rodrigues G, FeRREIRA J, FALCÃo F et al. Diagnosis of stroke by the nonneurologist. A validation study. Stroke 1998; 29: 1106-1109.

31. Allen CM. Clinical diagnosis of the acute stroke syndrome. Q J Med 1983; 52: 515-523.

32. WeISBERg LA, NicE CN. Intracranial tumors simulating the presentation of cerebrovascular syndromes. Early detection with cerebral computed tomography (CCT). Am J Med 1977; 63: 517-524.

33. Merino JG, Luby M, Benson RT, Davis LA, Hsia AW, Latour LL, ET AL. Predictors of Acute Stroke Mimics in 8187 Patients Referred to a Stroke Service. J Stroke Cerebrovasc Dis 2013; 22: 397-403.

34. Montaner J, Mendioroz M, Ribó M, Delgado P, Quintana M, Penalba A, Et al. A panel of biomarkers including caspase-3 and D-dimer may differentiate acute stroke from strokemimicking conditions in the emergency department. J Intern Med 2011; 270: 166-174. 